ARTICLE OPEN

Check for updates

\title{
Development of an optogenetic gene sensitive to daylight and its implications in vision restoration
}

Yoshito Watanabe ${ }^{1}$, Eriko Sugano (D) $^{1 凶}$, Kitako Tabata (iD) ${ }^{1}$, Akito Hatakeyama1', Tetsuya Sakajiri (D) ${ }^{2}$, Tomokazu Fukuda ${ }^{1}$, Taku Ozaki (iD) Tomoya Suzuki (iD), Tatsuki Sayama ${ }^{1}$ and Hiroshi Tomita (iD) ${ }^{1,3 凶}$

Optogenetic gene-mediated therapy for restoring vision is thought to be a useful treatment for blind patients. However, light sensitivity achieved using this gene therapy is inferior to that of daylight vision. To increase light sensitivity, we designed three mutants using a bioinformatics approach. Nucleotide sequences encoding two sites in the extracellular loops (ex1, ex3) of mVChR1 close to simulated ion-conducting pathways were replaced by homologous amino acid-encoding sequences of ChR1 or ChR2. The light sensitivity of ex3mV1 was higher than that of $\mathrm{mVChR1}$ at $405-617 \mathrm{~nm}$. Visual responses were restored in Royal College of Surgeons rats with genetically degenerating photoreceptor cells transfected with ex3mV1Co, wherein transmembrane of sixth (TM6) in ex3mV1 was additionally replaced with the corresponding domain of CoChR; these rats responded to light in the order of $\mu \mathrm{W} / \mathrm{mm}^{2}$. Thus, ex3mV1Co might be useful for the restoration of advanced visual function.

npj Regenerative Medicine (2021)6:64; https://doi.org/10.1038/s41536-021-00177-5

\section{INTRODUCTION}

The light-driven cation channel channelrhodopsin-2 (ChR2) plays a central role in optogenetics. Optogenetic techniques have been used in a variety of fields. In neuroscience studies, the ChR2 gene has been transfected into neuronal cells for the optical control of neural ${ }^{3}$ and behavioural ${ }^{4}$ activities and for performing studies on brain function ${ }^{5}$ and mechanisms of neuronal diseases $^{6,7}$. In the field of vision research, optogenetic techniques have been used to treat blindness ${ }^{8}$. Bi et al. ${ }^{9}$ and our group ${ }^{10-12}$ reported that the transfection of ChR2 into the retinal ganglion cells (RGCs) of blind mice and rats could recover their light responses. Safety studies have also been performed in rats ${ }^{13}$ and marmosets; ${ }^{14}$ continuous expressions of the ChR2 gene derived from the unicellular green alga Chlamydomonas reinhardtii did not result in any adverse effect caused by immunological responses. Currently, a few clinical trials (NCT02556736, NCT03326336 ${ }^{15}$ ) are underway for investigating the efficacy of gene therapies employing optogenetic genes for restoring vision.

Previously, we had developed modified Volvox channelrhodopsin1 (mVChR1), which has a broader range of wavelength sensitivities than that of ChR2 ${ }^{16}$. We found that the transfection of the $m V C h R 1$ gene into blind rats restored their VEPs upon stimulation at $450-600 \mathrm{~nm}$ and that optomotor responses were elicited with all colour stripes. We also found that the recovered visual responses were maintained over a year without any adverse effects ${ }^{17}$. However, unnaturally bright light intensities were required to elicit responses. We also reported that the dual expression of optogenetic genes in the retina expands the range of wavelength sensitivities but not that of light sensitivities ${ }^{18}$. This motivated us to develop a channelrhodopsin that is more photosensitive than mVChR1.

Various types of channelrhodopsins have been developed, such as ChR2 (E123T/T159C) ${ }^{19}$ and ChIEF (chimera) ${ }^{20}$. However, most of them have been evaluated in experiments that utilized light in the order of $\mathrm{mW} / \mathrm{mm}^{2}$ for stimulation. Because daylight is dimmer $\left(\mu \mathrm{W} / \mathrm{mm}^{2}\right.$ rather than $\left.\mathrm{mW} / \mathrm{mm}^{2}\right)$, channelrhodopsins should have higher light sensitivities or at least be responsive to light in the order of $\mu \mathrm{W} / \mathrm{mm}^{2}$ for restoring vision in blind patients.

Here, we report the development of multiple $\mu \mathrm{W} / \mathrm{mm}^{2}$ lightresponsive channelrhodopsins by the replacement of amino acids related to ion-conducting pathways in $\mathrm{mVChR} 1$ with corresponding ones from ChR2. We also characterized the photosensitivity of the modified channelrhodopsin ex3mV1Co by patch-clamp recording in the human embryonic kidney (HEK) 293 cells and in vivo experiments using genetically blind rats. Our newly developed optogenetic gene, with its higher and broad-spectrum light sensitivities, would be especially useful for gene therapy aimed at restoring daylight vision.

\section{RESULTS \\ Design of mutants by bioinformatics approaches}

lon-conducting pathways of ChR2 have been reported to be formed in TM1-3 and 7 structures ${ }^{21}$. The photocurrents of mVChR1, derived from VChR1, were lower than those of ChR2 ${ }^{16}$. The ion-conductive pathways of mVChR1 were simulated based on the crystal structure of $\mathrm{ChR2}^{21}$. Our previous research investigating the photocurrent properties of the chimeric protein of ChR1 with ChR2 showed that the chimaera including TM3 of ChR1 increased its photocurrents (Fig. 1a, grey-background box). We expected the TM3 of ChR1 to play a role in increased photocurrent elicitation, based on our previous research ${ }^{22}$. The TM3 of mVChR1 was replaced with the corresponding sequence of ChR1 because this sequence was included in the simulated ionconductive pathways of mVChR1 (Fig. 1b). The extracellular loop between TM2 and TM3 (ex1) and the extracellular loop between TM6 and TM7 (ex3) (Fig. 1c) were also identified at the entrance of the ion-conductive pathways.

\footnotetext{
${ }^{1}$ Graduate Course in Biological Sciences, Iwate University Division of Science and Engineering, 4-3-5 Ueda, Morioka, Iwate 020-8551, Japan. ${ }^{2}$ Department of Nutrition, Kyushu Nutrition Welfare University, 5-1-1 Shimoitozu, Kitakyushu Kokurakita-Ku, Fukuoka 803-0846, Japan. ${ }^{3}$ Clinical Research, Innovation and Education Center, Tohoku University Hospital, 1-1 Seiryo, Aoba, Sendai, Miyagi 980-8574, Japan. ${ }^{凶}$ email: sseriko@iwate-u.ac.jp; htomita@iwate-u.ac.jp
} 
a

C1C2

ChR1

ChR2

mVChR1

CoChR

ex1mV1

ex3mV1

ex3mV1Co

C1C2

ChR1

ChR2

mVChR1

GoChR

ex1mV1

ex3mV1

ex $3 \mathrm{mV} 1 \mathrm{Co}$
TM2

WEE IYVAT IEM IKFI IEYFHEFDEPAVIYSSNGNKTVWLRYAEWLLTCPVILIHL WEE IYVAT I EM IKF I IEYFHEFDEPAVIYSSNGNKTVWLRYAEWLLTCPVILIHL WEE IYVCA I EMVKVILEFFFEFKNPSMLYLATGHRVQWLRYAEWLLTCPVILIHL WEEVYVAL I EMMKSI IEAFHEFDSPATLWLSSGNGVVWMRYGEWLLTCPVLL I HL WEEVYVCCVELTKVI IEFFHEFDDPSMLYLANGHRVQWLRYAEWLLTCPVILIHL WEEVYVALIEMVKVILEFFFEFKEPAVIYSSNGNKTVWLRYAEWLLTCPVILIHL WEEVYVALIEMMKSI IEAFHEFDEPAVIYSSNGNKTVWLRYAEWLLTCPVILIHL WEEVYVALIEMMKSIIEAFHEFDEPAVIYSSNGNKTVWLRYAEWLLTCPVILIHL *** ** $* \begin{array}{lllll}* * * * * * & * & * & * * * * * * * * * * * & * * * *\end{array}$

\section{TM6}

\section{TM7}

RCRQVVTGMAWLFFVSWGMFP ILF ILGPEGFGVLSVYGSTVGHTI IDLMSKNCWGLLGHYLRVL I HEH I L IH I CRDLVRYLAWLYFCSWAMFPVLFLLGPEGFGH I NQFNSA I AHA I LDLASKNAWSMMGHFLRVK I HEH I LLY RCRQVVTGMAWLFFVSWGMFPILFI LGPEGFGVLSVYGSTVGHTI IDLMSKNCWGLLGHYLRVL I HEH I LIH I CRELVRVMAWTFFVAWGMFPVLFLLGTEGFGH ISPYGSA I GHS I LDL I AKNMWGVLGNYLRVK I HEH I LLY RPRTVVR I MAWLFFL SWGMFPVLFVVGPEGFDA I SVYGST I GHT I IDLMSKNCWGLLGHYLRVL I HQH I I I Y ICRELVRVMAWTFFVAWGMFPVLFLLGTEGFGHISPYGSAIGHSILDLI AKNMWGVLGNYLRVKIHEHILLY ICRELVRVMAWTFFVAWGMFPVLFLLGPEGFGVLSVYYGSAIGHSILDLIAKNMWGVLGNYLRVKIHEHILLY RPRTVVRIMAWLFFLSWGMFPVLFVVGPEGFGVLSV

$$
* * * * \quad * * * * * * * * * * * \frac{*}{*} * * * * * * \quad * * * * * * * *
$$

b

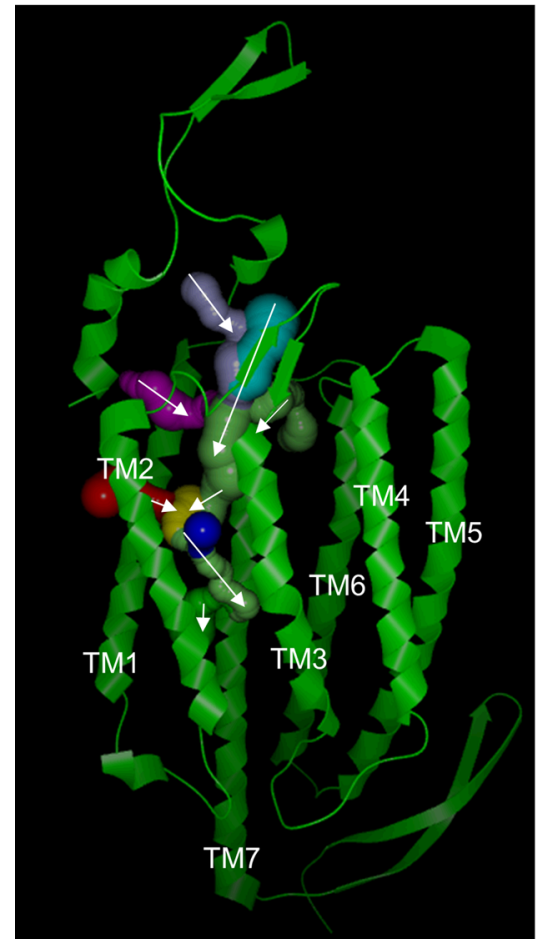

C

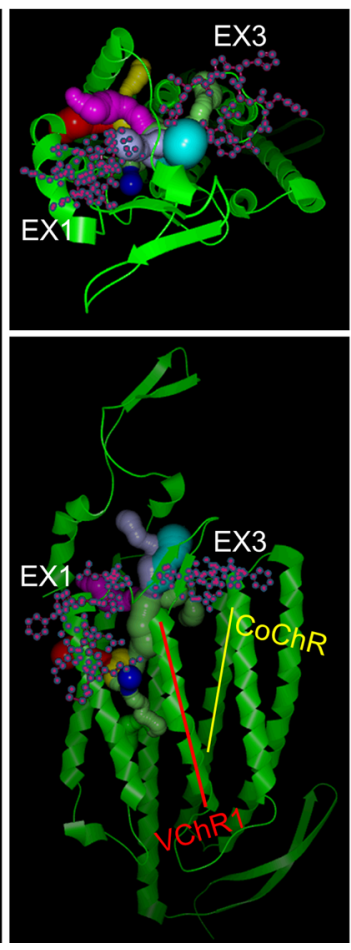

Fig. 1 Nucleotide sequences encoding extracellular regions close to the simulated ion-conducting pathways in mVChR1 were replaced with the corresponding sequences of Chlamydomonas-derived channelrhodopsins and Chloromonas oogama-derived channelrhodopsin. a The amino acid sequences around the extracellular loops of TM2 and TM3 and TM6 and TM7 are shown. The replaced amino acids from channelrhodopsin-1, channelrhodopsin-2 and CoChR are shown as grey, blue and red background boxes, respectively. The open boxes indicate amino acids of mVChR1 that were not replaced. b The ion-conductive pathways were simulated by CAVER (http://www.caver.cz/). Some simulated tunnels are shown by white arrows. $\mathbf{c}$ The replaced amino acids and the transmembrane domain are indicated by purple dots and red and yellow bars. Extracellular regions between TM2 and TM3 and TM6 and TM7 are abbreviated as ex1 and ex3, respectively.

\section{Photocurrents and kinetic profiles in three mutants}

The photocurrents from HEK293 cells transfected with the mutant genes, ex3mV1 or ex13mV1, described in the Methods and Fig. 1, were compared with those from mVChR1-expressing cells. The amplitudes of the photocurrents from ex3mV1-HEK293 cells were significantly $(p<0.001)$ larger than those from mVChR1-HEK293 cells (Fig. 2b). The turning-ON (TON; Fig. 2c) and turning-OFF (TOFF; Fig. 2d) durations of ex3mV1- and ex13mV1-HEK293 cells were significantly longer than those of mVChR1-HEK293 cells.
Analysis of the stimulus duration response revealed that the photocurrents of ex3mV1-HEK293 cells were larger for all stimulus durations compared to those of the mVChR1-expressing cells (Fig. $2 e)$. In addition, the photocurrents of the cells transfected with ex $2 m V 1$, wherein the ex2 amino acid-encoding sequence was replaced with the corresponding one from ChR2, showed no significant difference compared with those of mVChR1-HEK293 cells (Fig. 2a and Supplementary Fig. 1). Venus fluorescence, indicating the expression of each gene, was found in similar locations within the HEK cells (Fig. 2f). 
a

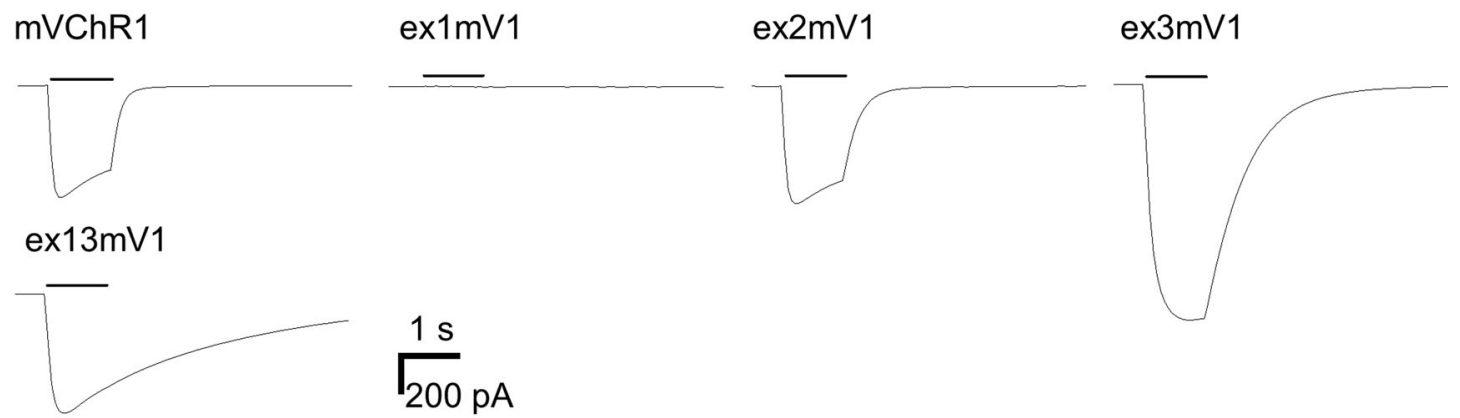

b

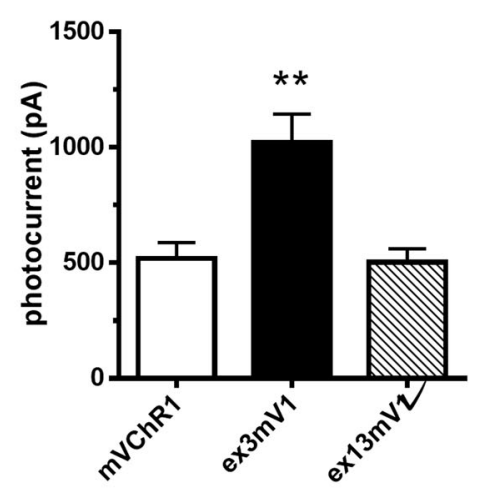

C

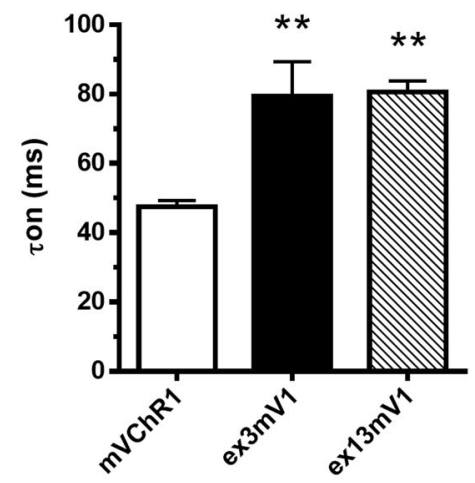

d

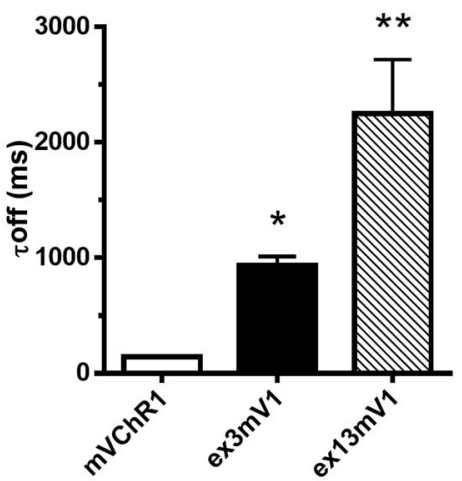

e

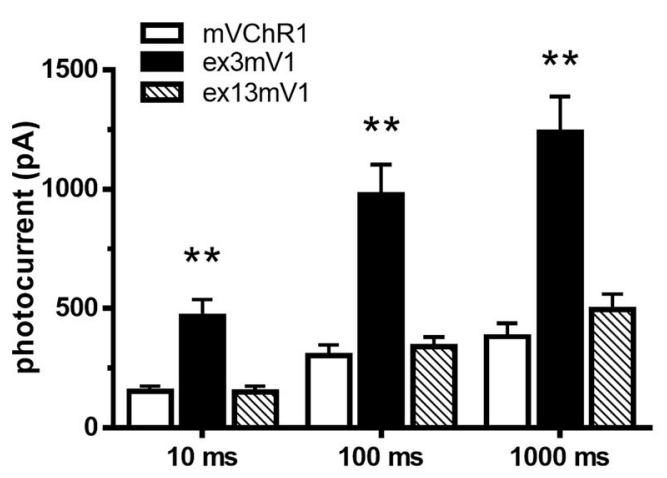

f

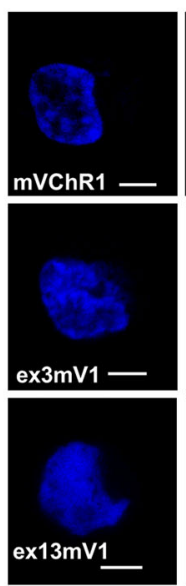

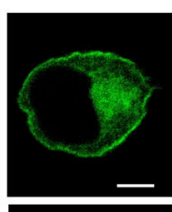
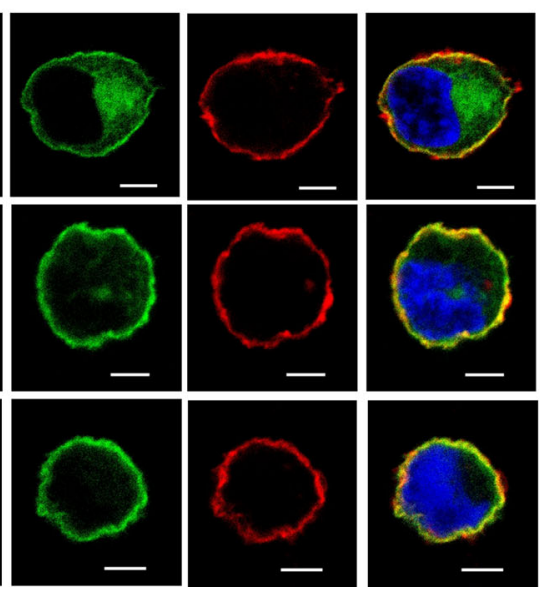

Fig. 2 Kinetic profiles of photocurrents in cells expressing mVChR1, ex3mVChR1 and ex13mVChR1 genes. a Typical waveforms recorded from each gene-expressing cell with a stimulus of light at $505 \mathrm{~nm}$ for $1000 \mathrm{~ms}$ at $1 \mu \mathrm{W} / \mathrm{mm}^{2}$. b Comparison of peak photocurrents stimulated at $505 \mathrm{~nm}$ for $10 \mathrm{~s}$. c, d Differences in the turning-ON $(\tau \mathrm{ON} ; \mathrm{c})$ and turning-OFF constants $(\tau \mathrm{OFF}$; d) in cells expressing different genes, with a stimulus of light at $505 \mathrm{~nm}$ for $1000 \mathrm{~ms}$ at $1 \mu \mathrm{W} / \mathrm{mm}^{2}$. e Photocurrents elicited using various durations of light stimulus with $505 \mathrm{~nm}$ at $1 \mu \mathrm{W} /$ $\mathrm{mm}^{2}$. f There was no difference between the protein localisation of each expressed gene. Bar $=5 \mu \mathrm{m}$. The numbers of $\mathrm{mVChR1}$, ex3mVChR1 and ex13mVChR1 cells recorded in each graph from $\mathrm{b}$ to e were 11,8 and 6 , respectively. Data are presented as mean \pm SEM $\left({ }^{*} p<0.05,{ }^{* *} p<\right.$ 0.01; Dunnett's multiple comparison test).

\section{Photocurrents in ex3mV1Co-expressing cells}

The photocurrent of ex3mV1Co, which was obtained by modifying TM6 and the C-terminal of ex3mV1, was also investigated. Typical waveforms for this photocurrent are shown in Fig. 3a, b. The photocurrent of ex3mV1Co was significantly larger than that of $\mathrm{mVChR} 1$ (Fig. 3c), and there was no significant difference in the TON between mVChR1-HEK293 cells and ex3mV1Co-HEK293 cells (Fig. 3d). The TOFF of ex3mV1CoHEK293 cells was longer than that of mVChR1-HEK293 cells (Fig. 3e). Comparison of ex3mV1-HEK293 cells (Fig. 2a-c) and 
a

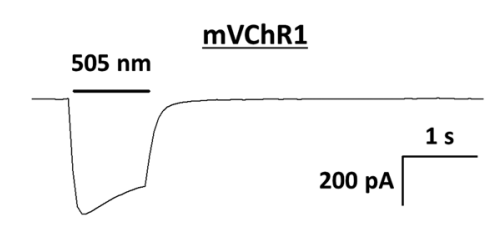

b

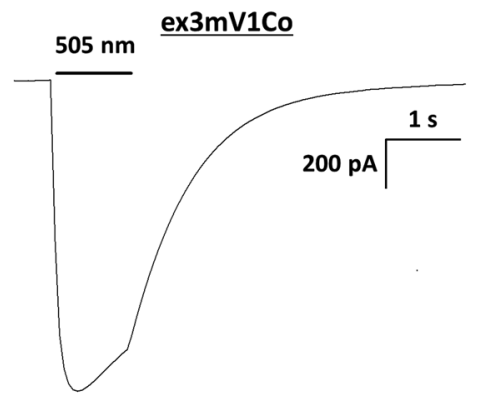

e

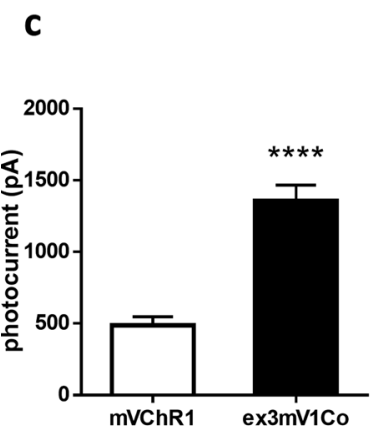

d

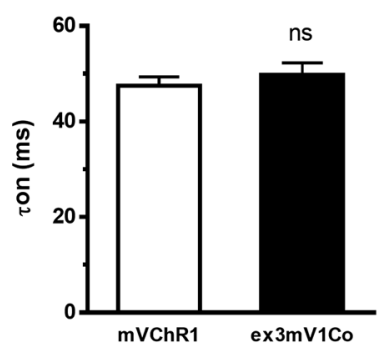

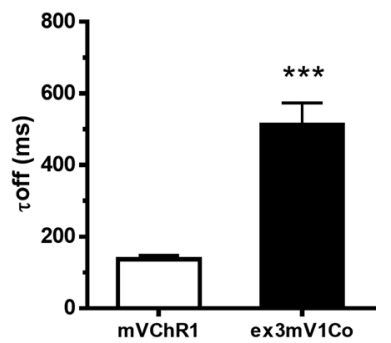

f

membrane Voltage $(\mathrm{mV})$

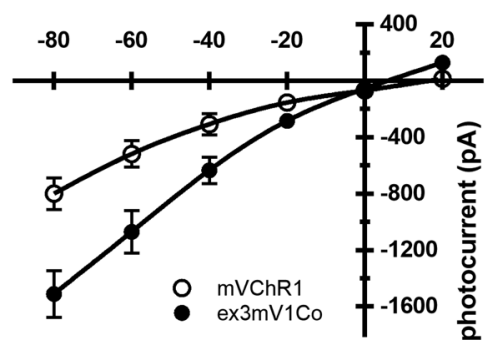

Fig. 3 Comparison of kinetic profiles of photocurrents elicited from mVChR1- and ex3mV1Co- expressing cells. a, b Typical waveforms of cells expressing the mVChR1 or ex3mV1Co gene stimulated at $505 \mathrm{~nm}$. c Comparison of peak photocurrents stimulated at $505 \mathrm{~nm}$ in mVChR1and ex3mV1Co-expressing cells (mVChR1: $n=8$, ex3mV1Co: $n=11$ ). d, e Differences in the turning-ON and turning-OFF constants ( $\tau$ ON; $d$, $\tau$ OFF; e) in each cell with a light stimulus of $505 \mathrm{~nm}$ (mVChR1: $n=8$, ex3mV1Co: $n=11)$. $f-V$ curves of mVChR1 and ex3mV1Co are presented (mVChR1: $n=6$, ex3mV1Co: $n=5$ ). The stimulus intensities for all recordings were applied for $1 \mu \mathrm{W} / \mathrm{mm}^{2}$, and the stimulus durations were set at $1 \mathrm{~s}$, except for the $\tau \mathrm{ON}$ - and $\tau \mathrm{OFF}$-analyses. The stimulus duration of the $\tau \mathrm{ON}$ - and $\tau$ OFF-analyses were set at $10 \mathrm{~s}$. Data are presented as the mean \pm SEM ${ }^{* * *} p<0.001,{ }^{* * * *} p<0.0001$; unpaired $t$ test).

ex3mV1Co-HEK293 cells (Fig. 3c-e) showed that the replacement of TM6 and the C-terminal region affected the TON of ex3mV1. There were a slight difference in the current-voltage $(I-V)$ curves between mVChR1-HEK293 and ex3mV1Co-HEK293 cells (Fig. 3f). The reversal potential ( $x$-axis intersection) of ex3mV1Co-HEK293 cells was slightly shifted toward $0 \mathrm{mV}$, compared to that of mVChR1-HEK293 cells.

We also investigated the wavelength sensitivities of ex3mV1Co. The peak photocurrents of mVChR1-HEK293 cells and ex3mV1Co-HEK293 cells were maximum at $505 \mathrm{~nm}$, and the peak (Fig. 4a, $p<0.0001$ ) and light-off photocurrents (Fig. $4 b, p$ $<0.0001)$ of ex3mV1Co-HEK293 cells were significantly larger than those of mVChR1-HEK293 at $405-617 \mathrm{~nm}$. The rates of decay of ex3mV1Co-HEK293 cells were significantly lower than those of mVChR1-HEK293 cells (Fig. 4c) at 405-656 nm. The decay rate of mVChR1-HEK293 cells was maximum at $505 \mathrm{~nm}$, similar to that of ex3mV1Co-HEK293 cells. The light intensity responses and stimulus duration dependencies are shown in Fig. $4 d, e$, respectively. Even at light stimulus intensities of $0.04 \mu \mathrm{W} /$ $\mathrm{mm}^{2}$, a photocurrent was clearly elicited from ex3mVC1CoHEK293 cells. At a stimulus intensity of $0.2 \mu \mathrm{W} / \mathrm{mm}^{2}$, the photocurrent elicited from ex3mV1Co-HEK293 cells for a 10 or $100 \mathrm{~ms}$ stimulus duration was almost the same as that elicited from mVChR1-HEK293 cells with a stimulus intensity of $1.00 \mu \mathrm{W} /$ $\mathrm{mm}^{2}$ (Fig. 4d). Moreover, with an increase in stimulus duration, the difference in the photocurrents between ex3mV1Co-HEK293 and mVChR1-HEK293 cells became larger. The slope of ex3mV1Co-HEK293 cells was significantly steeper than that of mVChR1-HEK293 cells at 10-1000 ms (Fig. 4e, one-way ANCOVA test, $p<0.001$ ). All comparisons between mVChR1-HEK293 and ex3mV1Co-293 cells revealed statistically significant differences $(p<0.01)$. 
a

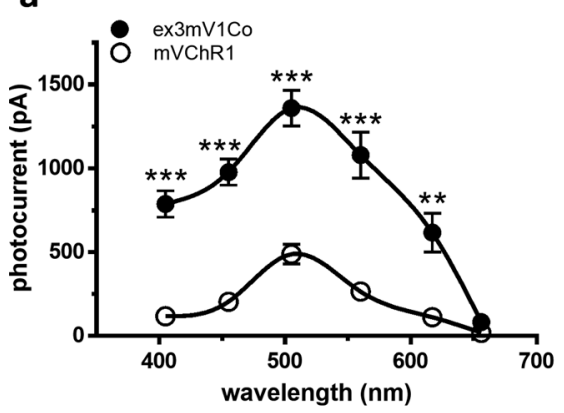

C

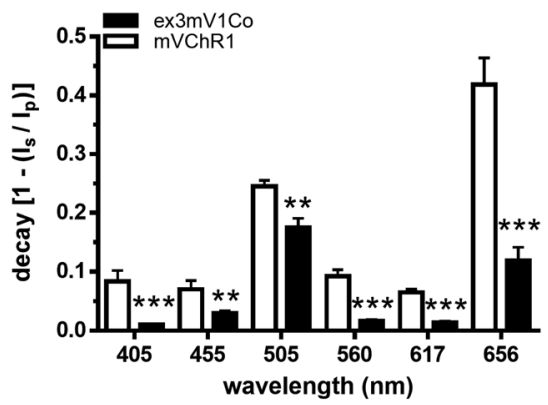

d

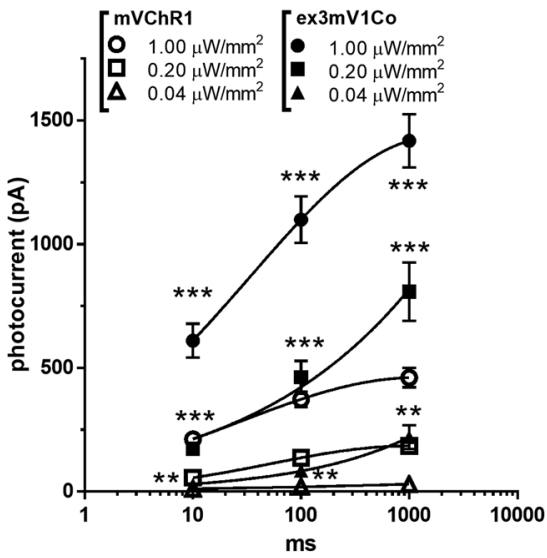

b

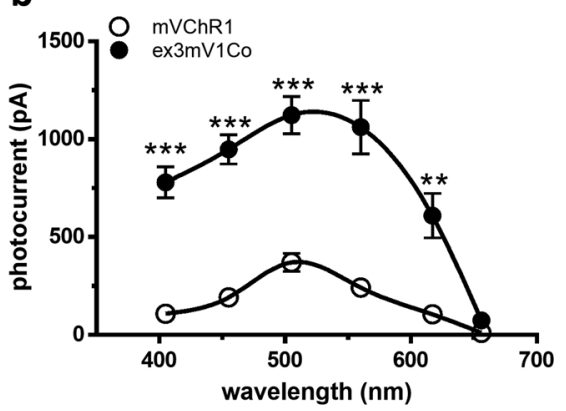

e

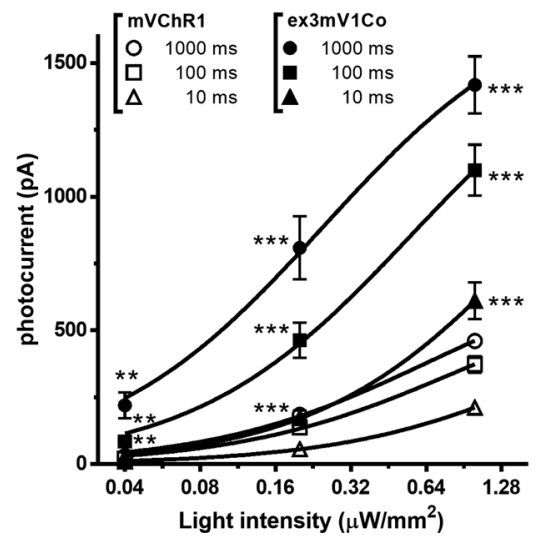

Fig. 4 Wavelength sensitivity and intensity responses in mVChR1- and ex3mV1Co-expressing cells. a, b Each peak (a) and light-off photocurrent (b) were measured under the exposure of various wavelengths of light $(405,455,505,560,617$ and $656 \mathrm{~nm})$ for $1 \mathrm{~s}$ (mVChR1: $n=8$, ex3mV1Co: $n=11 ;{ }^{*} p<0.05,{ }^{* *} p<0.01,{ }^{* * *} p<0.001$; Tukey's multiple comparison test). c Decay rates calculated from the data of the peak and light-off photocurrents. Data are presented as mean $\pm \operatorname{SEM}\left({ }^{*} p<0.05,{ }^{* *} p<0.01,{ }^{* * *} p<0.001\right.$, unpaired $t$ test). d Photocurrents were recorded for various stimulus intensities $\left(0.04,0.20\right.$ and $\left.1.00 \mu \mathrm{W} / \mathrm{mm}^{2}\right)$ and durations $(10,100$ and $1000 \mathrm{~ms})(n=8-9)$ with LEDs of $505 \mathrm{~nm}$.

\section{Adeno-associated virus-mediated ex3mV1Co gene transfection into genetically blind rats}

Adeno-associated virus (AAV)-mediated transfection of ex3mV1Co resulted in Venus fluorescence in the entire retina (Fig. 5a); however, the inferior part of the retina seemed to show better transfection efficiency. Most of the Venus fluorescence overlapped with rhodamine, indicating NeuN immunoreactivity (Fig. 5b). The 3-D image also showed co-labelling in an area on the retinal surface (Fig. 5c). The vertical section clearly indicated that ex3mV1Co was mainly expressed in the RGCs and the inner plexiform layer (Fig. 5d).

\section{Recording of visually evoked potentials (VEP)}

Royal College of Surgeons (RCS) rats (age, 7-10 months), which are genetically blind, were used for the experiments; they showed no response in visually evoked potentials (VEPs) (Fig. 6a). However, 2 months after transfection of the ex3mV1Co gene, VEPs could be clearly recorded with stimuli of 465, 525 and $650 \mathrm{~nm}$ (Fig. 6a, b), even with a stimulus intensity of $3.5 \mu \mathrm{W} / \mathrm{mm}^{2}$. VEPs could be recorded even 17 months after the transfection, indicating the maintenance of the recovered visual function for a long duration (Supplementary Figure 2). We also measured the response to light flashes repeated at different frequencies (Fig. 6c), which indicated no decrease in responses.

\section{Behavioural tests}

All of the ex3mV1CO-expressing rats were tracked with the range of spatial frequencies from 0.09 to 0.42 at the blue-black stripes (Fig. 6d). However, one among five rats could not track the spatial frequency at 0.36 of white-black or green-black stripes. In addition, one among five rats could not track the spatial frequency at 0.18 of red-black stripes. 

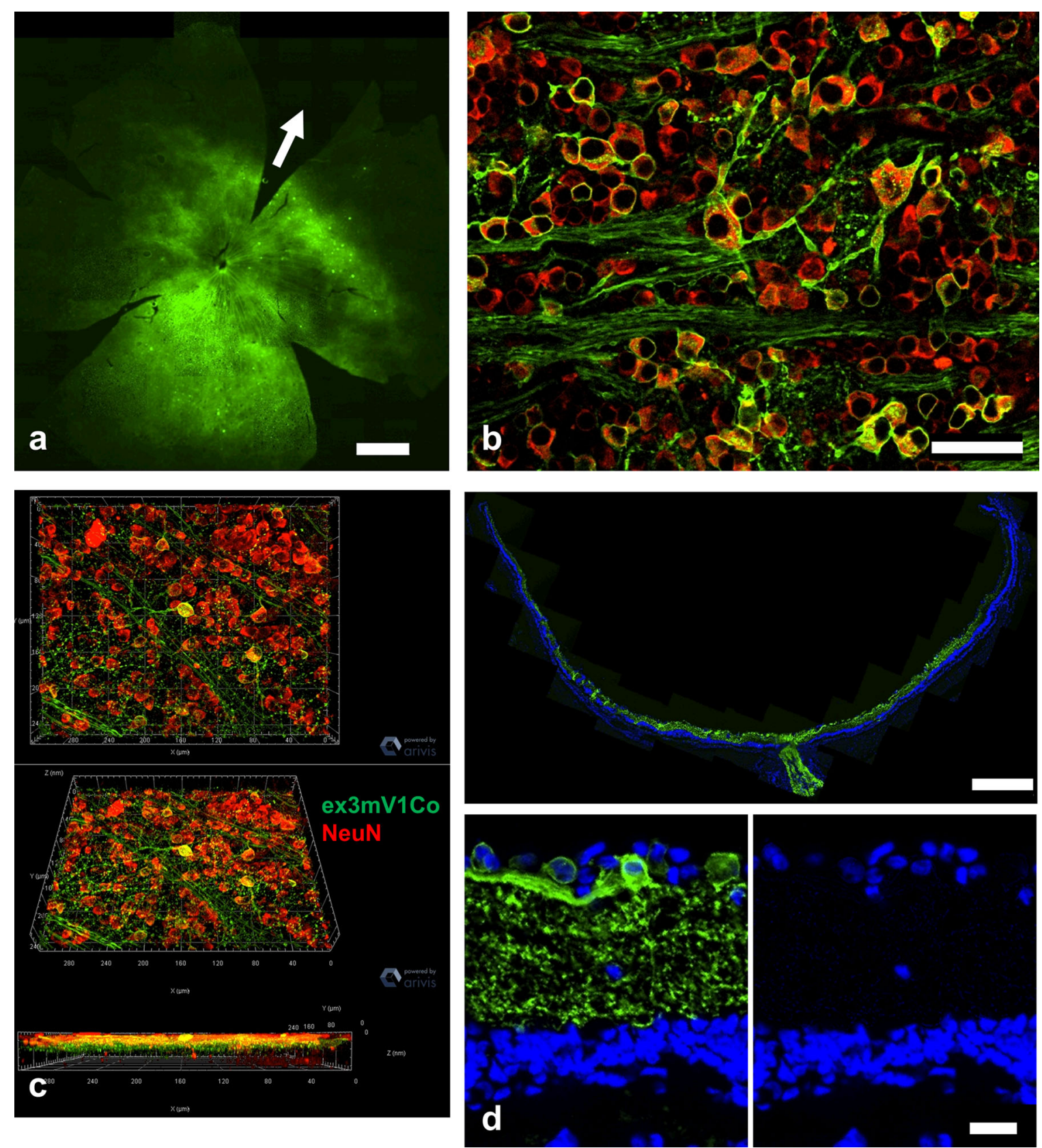

Fig. 5 AAV-mediated ex3mV1Co gene transfection into blind rat retinas. a Venus fluorescence (green) in the whole-mounted retina. The arrow indicates the 12 o'clock direction. Bar $=1000 \mu \mathrm{m}$. b The retinal ganglion cells of whole-mounted retinas were identified by immunostaining with the anti-NeuN antibody (red). Bar $=50 \mu \mathrm{m}$. c The 3-D view of the immunostained retinas. d Cryo-retinal section. Nuclear staining (blue) was performed using mounting media, including 4',6-diamidino-2-phenylindole (DAPI). Bar = $500 \mu \mathrm{m}$ (upper), $20 \mu \mathrm{m}$ (lower).

\section{DISCUSSION}

In this study, we modified the mVChR1 gene that we had previously synthesised using a bioinformatics approach, focusing on the ion-conductive pathways to improve light sensitivity. We found that the extracellular loop between TM6 and TM7 and TM6 play an important role in the photocurrent amplitude and channel kinetics, respectively. We also demonstrated that blind rats transfected with the ex $3 \mathrm{mV} 1 \mathrm{Co}$ gene could respond to irradiances measuring $\mu \mathrm{W} / \mathrm{mm}^{2}$.

The replacement of ex 1 unexpectedly resulted in a current loss in ex1mV1-expressing cells (Fig. 2a), whereas the replacement of ex3 produced a marked increase in current amplitude in ex1mV1expressing cells (Fig. 2b). Surprisingly, ex13mV1, including both ex1 and ex3, produced photocurrents with amplitudes similar to those of mVChR1. The representatives of channel kinetics, such as the rates of TON and TOFF, were reduced for ex $3 \mathrm{mV} 1$ and ex13mV1 compared to those for mVChR1 (Fig. 2c, d). These results indicated that ex 1 and ex3 (Fig. 1) played an important role in the light-induced ion channel activities of $\mathrm{mVChR} 1$. The contributions of ex 1 and ex3 to the photocurrent and channel kinetics are still unclear, but the fact that the loss of function caused by the replacement of ex 1 was restored by the additional replacement of ex3 might indicate that the interactions between ex 1 and neighbouring amino acids are related to its channel function.

To improve the channel kinetics, various mutants, such as chimeric channelrhodopsins and channelrhodopsin with single 
a

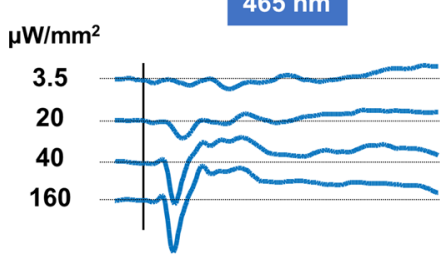

$\underset{20 \mathrm{msec}}{\stackrel{4}{100} \mu \mathrm{V}}$
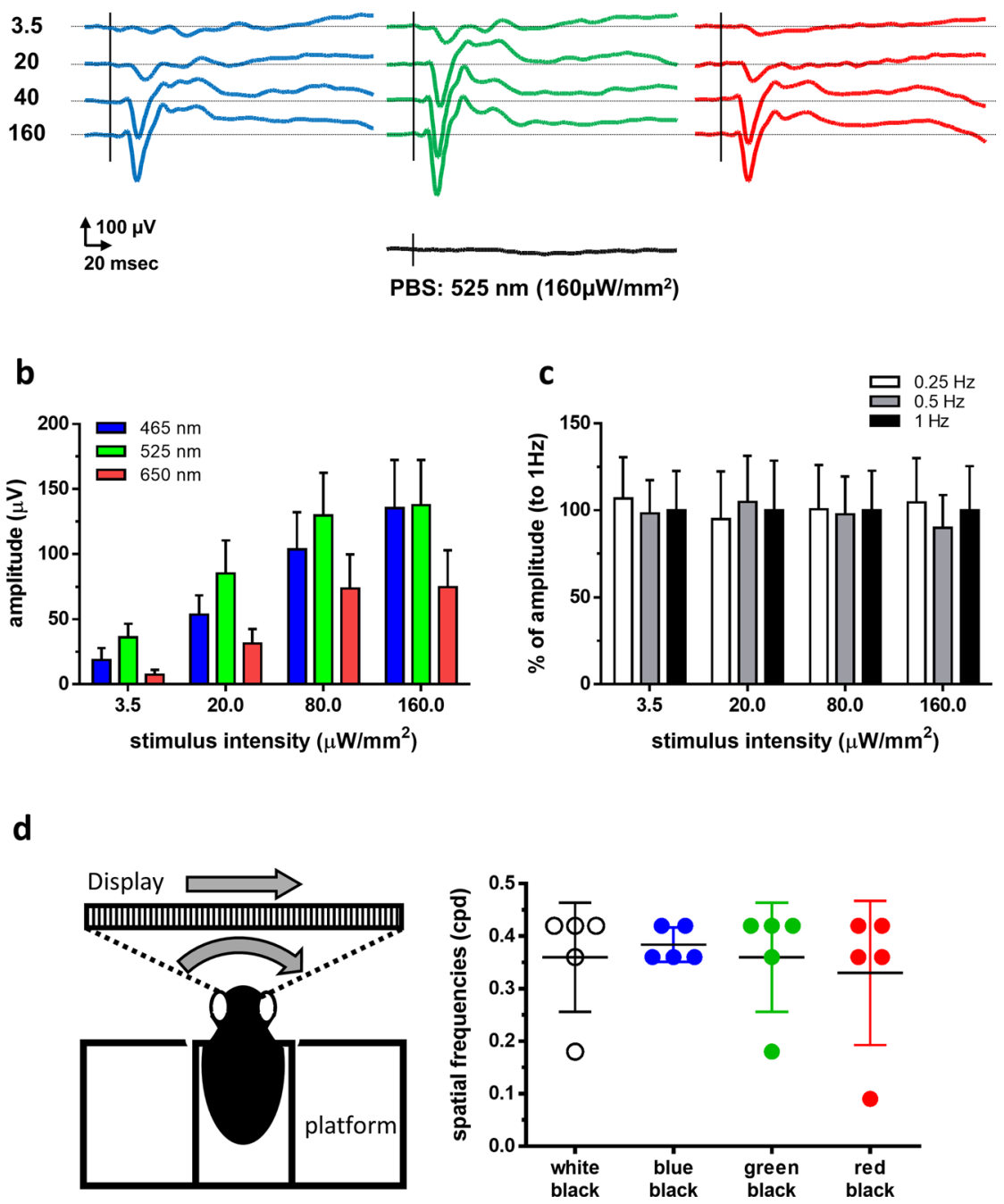

Fig. 6 Recordings of VEPs and behavioural assessments in ex3mV1Co-transfected rats. a Typical waveforms of VEPs with stimuli at blue $(465 \mathrm{~nm})$, green $(525 \mathrm{~nm})$ and red $(650 \mathrm{~nm})$ LEDs. b Evoked potentials in response to each LED with various intensities $(n=8)$. c Effects of stimulus frequencies on the evoked potentials $(n=7)$. Data are presented as mean \pm SEM. d Optomotor responses against white-black, blueblack, green-black and red-black stripes with various frequencies displayed on the screen $(n=5)$. Data are presented as mean \pm SD.

amino acid substitutions, have been reported ${ }^{23}$. In addition, the 3-D structure of the protein has been reported to affect its interaction with nearby transmembrane domains, which subsequently affects the channel characteristics ${ }^{24}$. We additionally modified ex3mV1 to improve the kinetics (such as tON and TOFF) of ex3mV1. CoChR has been reported to have fast kinetics and good membrane trafficking ${ }^{25,26}$. Ganjawala et al. ${ }^{27}$ also reported some positions of amino acid residues having an important role in the kinetics of the off-rate in CoChR. Meanwhile, we focused on the TM6 of CoChR, which is expected to affect the CoChR protein structure owing to the presence of retinal binding sites (corresponding to W261 of mVChR1) and was introduced into ex3mV1. Although the TON and TOFF rates of ex3mV1Co became shorter than those of ex3mV1, the TOFF of ex3mV1Co was not as short as that of mVChR1 (Figs. 2b, c and 3d, e); the mechanism underlying this phenomenon remains unclear. The molecular basis of photocurrent kinetic profiles has been explained using multiple-photocycle models ${ }^{28,29}$ defined as the transition rates, such as the closing state and the opening state $e^{30,31}$. In the present study, we could not elucidate the photocycle because of the use of the whole-cell patch-clamp. Further studies using singlechannel recordings such as the patch-clamp methods of cellattached, inside-out or outside-out mode are needed to clarify the photocycle model of ex3mV1Co.

The peak photocurrent of ex3mV1Co was increased compared to that of ex3mV1, and the opening speed of ex3mV1Co remained the same as that of mVChR1, but the closing speed of ex3mV1Co was slower than that of $m V C h R 1$. The difference in the primary amino acid structures between ex $3 \mathrm{mV} 1$ and ex $3 \mathrm{mVCo}$ is mainly TM6. The position of proline ${ }^{246}$ in the TM6 of ex3mV1Co-the corresponding amino acid of which is cysteine ${ }^{246}$ in ex3mV1-is related to the ion-conductive pathway and forms the ion tunnel with valine ${ }^{241}$ in TM5 and isoleucine ${ }^{309}$ in TM7, as revealed by a CAVER simulation (Supplementary Fig. 3a, b). The diameters of the ion tunnel at position 246 in ex3mV1Co and ex3mV1 were 1.33 and $1.42 \AA$, respectively. This difference in diameters did not seem to result in a difference in kinetics. The average distance between the amino acid at position 246 and $\mathrm{I}^{309}$ of ex3mV1Co was longer than that between the amino acid at position 246 and ${ }^{309}$ of ex3mV1, although the average distance between the amino acid 
at position 246 and valine ${ }^{241}$ was almost the same in the molecular dynamics simulation (Supplementary Fig. 4a). The longer distance between the amino acid at position 246 and $\mathrm{I}^{309}$ of ex3mV1Co, owing to the replacement of TM6 [cystei$\mathrm{ne}^{246}(\mathrm{ex} 3 \mathrm{mV} 1)$ to proline $\left.{ }^{246}(\mathrm{ex} 3 \mathrm{mV} 1 \mathrm{Co})\right]$, is expected to be one of the factors contributing to its increased photocurrent. In addition, the average potential energy between the amino acid at position 246 and $\mathrm{I}^{309}$ of ex3mV1Co was lower than that of ex3mV1 (Supplementary Fig. 4b). The faster opening kinetics of ex3mV1Co than that of ex3mV1 might be due to the weaker interaction of proline $^{246}$ with isoleucine ${ }^{309}$ in ex $3 \mathrm{mV} 1$ Co than that in ex3mV1, and its decreased potential energy might affect its photosensitivity.

The reversal potential, one of the indications for the ion selectivity, of ex3mV1Co has shifted about $15 \mathrm{mV}$ to the negative direction compared to that of $\mathrm{mVChR1}$. The equilibrium potential of sodium ions is approximately $+60 \mathrm{mV}$. The reversal potential is close to $+60 \mathrm{mV}$ for highly sodium ion-selective channels $\mathrm{s}^{32}$. In addition, the reversal potential shifts in the negative direction in channels when other cations, such as protons and potassium ions, are also permeable 33,34 . The negative shift of the reversal potential may be due to a change in ion selectivity-for example, a lower sodium-selectivity in ex3mV1Co than in mVChR1.

In cation channelrhodopsin, $\mathrm{ChR} 2$, the amino acid residues in the transmembrane domain has an important role in ion selectivity ${ }^{35,36}$. There is no report that the key residue related to the ion selectivity is in the extracellular loop. However, in anion channelrhodopsin, the amino acid residue R84, the extracellularloop amino acid residue R84 helps determine anion permeability ${ }^{37,38}$. Therefore, our data indicated that amino acids at ex3, the extracellular loop located between TM6 and TM7 of ex3mV1Co, in ex3mV1Co might be involved in sodium-ion selectivity.

Gene therapy using ChRs that target surviving neurons, RGCs, or bipolar cells is expected to be a promising strategy for restoring vision to blind patients. We aimed to determine whether ex3mV1Co can function in a rat model of retinal degeneration. It was confirmed that ex3mV1Co-Venus was expressed in vivo and that the maximum photosensitivity of ex $3 \mathrm{mV} 1 \mathrm{Co}$ was observed in the presence of green light, as observed in vitro. In addition, the developed ex3mV1Co exhibited broader wavelength sensitivities towards blue, green, and red light (Fig. 6). Therefore, ex3mV1Co can also function in vivo. More importantly, rats transfected with the ex3mV1Co gene acquired the function of responding to light in the order of $\mu \mathrm{W} / \mathrm{mm}^{2}$. Behavioural studies also demonstrated that rats could respond to moving stripes presented on a liquid crystal display, although the responses differed with the colour displayed. The recovered visual function obtained by gene therapy would likely be highly dependent on the gene transfection into the retina ${ }^{39}$. Therefore, transfection of ex3mV1Co could be a potent technique to restore advanced visual function. However, a well-organised visual system like human vision recognises even a single photon ${ }^{40}$. The threshold response to light in ex3mV1Co was $0.04 \mu \mathrm{W} / \mathrm{mm}^{2}$ (Fig. 4e), which corresponds to approximately $10^{11}$ photons $/ \mathrm{mm}^{2}$. Thus, even though ex3mV1Co shows remarkably high light sensitivity compared to other optogenetic proteins, its light sensitivity is still considerably different from that of natural vision. Therefore, there might be a need to use a combination of other mechanical devices such as a goggle apparatus or other optogenetic genes with ex3mV1Cogene therapy so that patients might achieve visual function comparable to that of natural vision.

We found that the third extracellular loop was implicated in the selectivity of sodium ions and that the TM6 and C-terminal regions played some roles in channel kinetics. Our findings contribute to the development of novel optogenetic genes that may be more sensitive to light and exhibit different kinetic profiles. However, currently, there is no information that might allow the control of wavelength sensitivities. Further study is needed to identify the amino acid sequences that determine wavelength sensitivities.

\section{METHODS}

\section{Design of mutants and plasmid preparations}

To improve ion permeability, TM3 and the nucleotide sequences encoding three sites (ex1, ex2 and ex3) close to the extracellular loops in mVChR1 were replaced with those encoding the corresponding sites of ChR1 and ChR2, respectively. TM3 of ChR1 (Fig. 1a) was expected to contain the amino acid sequence related to conductance ${ }^{22}$, and the ex 1 and ex 3 sites were considered to be associated with the ion-conducting pathways, as indicated by the CAVER 3.0 simulation $^{41}$ (Fig. 1b) based on the previously reported crystal structure of $\mathrm{C1C}_{1}{ }^{42}$. The genes encoding the amino acid sequences of the ex 1 and ex 3 sites in mVChR1 were replaced with the corresponding sequences in ChR2 and named ex $1 \mathrm{mV} 1$ and ex $3 \mathrm{mV} 1$, respectively, and the gene containing replacements for nucleotide sequences encoding both sites was named ex13mV1 (Fig. 1c). Further, the sequence encoding TM6 and the C-terminal region of ex $3 \mathrm{mV} 1$ was replaced with the corresponding sequence of Chloromonas oogamaderived channelrhodopsin $(\mathrm{CoChR})^{43}$ to form ex3mV1Co.

The plasmid vectors containing each gene were designed as previously described ${ }^{44}$. In brief, the $\mathrm{N}$-terminal fragment of each gene was fused to Venus fluorescent protein in frame at the end of each gene-coding fragment. The Venus-fused cDNAs were then subcloned into the EcoRI and BamHI sites of the 6P1-CAG plasmid ${ }^{12}$. These plasmid vectors were used for transfecting the respective genes into HEK293 cells and for preparing the AAV type 2 vector.

\section{Cell preparation}

HEK293 cells (RCB1637: Riken Bioresource Center, Tsukuba, Japan) were cultured in a minimum essential medium (Thermo Fisher Scientific, Tokyo, Japan) supplemented with $10 \%$ foetal bovine serum under a $5 \% \mathrm{CO}_{2}$ atmosphere at $37{ }^{\circ} \mathrm{C}$. The culture medium was changed every 3 days, and cells were passaged at $80 \%$ confluence using a $0.02 \%$ ethylenediaminetetraacetic acid (Sigma-Aldrich, St. Louis, MO)/DPBS solution.

\section{Gene transfection into HEK293 cells}

HEK293 cells were transfected with the pAAV type 2 plasmid vectors (Agilent Technology, Tokyo, Japan) containing a CAG promoter using a slight modification of the calcium phosphate method ${ }^{45}$. In brief, cells were seeded onto the $60 \mathrm{~mm}$ culture dish at a concentration of $2.5 \times 10^{6}$ cells 1 day before the transfection, and the culture medium was replaced before transfection. The calcium phosphate-DNA particles using $6 \mu \mathrm{g}$ of plasmid DNA were then added to the $60 \mathrm{~mm}$ dish, and the medium was replaced with fresh culture medium following incubation in a $5 \% \mathrm{CO}_{2}$ atmosphere at $37^{\circ} \mathrm{C}$ for $6 \mathrm{~h}$. The cells were incubated in a $5 \% \mathrm{CO}_{2}$ atmosphere at $37^{\circ} \mathrm{C}$ for $24-30 \mathrm{~h}$ and were then seeded onto glass slides 1 day before the patch-clamp recordings were performed.

\section{Patch-clamp recordings}

Whole-cell patch-clamp recordings were performed using cultured HEK293 cells 2 days post transfection. Photocurrents were elicited by stimuli of various wavelengths $(405,455,505,560,617$ and $656 \mathrm{~nm})$ from light-emitting diodes (Mightex Systems, Pleasanton, CA, USA) and recorded using an EPC-10 amplifier (HEKA Electronik, Lambrecht, Germany) in whole-cell patch-clamp mode. The intensity of light at each wavelength was adjusted to $1 \mu \mathrm{W} / \mathrm{mm}^{2}$ by setting an appropriate current for each wavelength. The data were collected by filtering at $10 \mathrm{kHz}$ and sampling at $5 \mathrm{kHz}$. The photocurrent was measured as the increase in inward current, and series resistance compensation was applied at $70 \%$. The internal solution contained $130 \mathrm{mM} \mathrm{CsCl}, 10 \mathrm{mM}$ HEPES, $2 \mathrm{mM} \mathrm{MgCl}_{2}, 0.1 \mathrm{mM}$ $\mathrm{CaCl}_{2}, 10 \mathrm{mM} \mathrm{NaCl}, 2 \mathrm{mM} \mathrm{Na}$ ATP and $1.1 \mathrm{mM}$ EGTA, with the pH adjusted to 7.2. The external solution contained $138 \mathrm{mM} \mathrm{NaCl}, 3 \mathrm{mM} \mathrm{KCl}, 1 \mathrm{mM}$ $\mathrm{CaCl}_{2}, 2 \mathrm{mM} \mathrm{MgCl}, 4 \mathrm{mM} \mathrm{NaOH}$ and $10 \mathrm{mM}$ HEPES, with the $\mathrm{pH}$ adjusted to 7.4 by $\mathrm{HCl}$. The TON and TOFF time constants were analysed as previously described ${ }^{22,46}$ (Supplementary Fig. 5). In brief, TON was measured as the time to reach $1-\mathrm{e}^{-1}(63 \%)$ of the peak amplitude at the maximal photocurrent during exposure to the light stimulus. The TOFF was measured as the time to reach $\mathrm{e}^{-1}(37 \%)$ of the light-off amplitude at the end of exposure to the light stimulus. The decay was quantified as the 
difference between the peak and light-off amplitudes divided by the peak amplitude.

\section{Animals}

All animal experiments were conducted in accordance with the guidelines proposed by the Animal Care and Use Committee of Iwate University, Japan (approval No. A201801). The experiments were conducted on 7-10month-old Royal College of Surgeons (RCS; rdy/rdy) ${ }^{47}$ rats obtained from CLEA Japan (Tokyo, Japan). The rats were housed under conditions of cyclic light (On: 8:00 AM, 200 lux; Off: 8:00 PM) at $23^{\circ} \mathrm{C} \pm 1{ }^{\circ} \mathrm{C}$ and provided ad libitum access to laboratory chow and water. The average weights of the rats were $250.686 \mathrm{~g}$ (Fig. 6b), $278.5 \mathrm{~g}$ (Fig. 6c) and $535.44 \mathrm{~g}$ (Fig. 6d).

\section{Preparation of the AAV vector}

Plasmid vectors containing ex $3 \mathrm{mV} 1 \mathrm{Co}$ were used to produce the AAV type 2 vector. The AAV Helper-Free System (Agilent Technologies, Santa Clara, CA, USA) was used to produce infectious AAV virions, according to the manufacturer's instructions. AAV vectors were purified using a previously described the single-step column purification method ${ }^{48}$. The concentration of the purified AAV vectors was determined by measuring the levels of the AAV capsid protein using ELISA (PROGEN, Heidelberg, Germany).

\section{Intravitreal injection of AAV}

Rats were anaesthetised by intramuscular injection of $35 \mathrm{mg} \mathrm{kg}^{-1}$ ketamine and $3.5 \mathrm{mg} \mathrm{kg}^{-1}$ xylazine. Under an operating microscope, $5 \mu \mathrm{L}$ of a suspension containing $1 \times 10^{12}$ virions $\mathrm{mL}^{-1}$ was intravitreally injected through the ora Serrata using an automatic syringe (Neurosyringe AC; ACrux Inc., Morioka, Japan) with a 32-gauge needle (Hamilton Company, Reno, NV, USA).

\section{Recordings of VEPs}

Two months after the intravitreous injections, VEPs were recorded as described previously ${ }^{16}$. Briefly, at least seven days before the recordings, the electrodes were implanted epidurally $6.8 \mathrm{~mm}$ behind the bregma and $3 \mathrm{~mm}$ lateral to the midline of both hemispheres. A reference electrode was implanted epidurally on the midline $12 \mathrm{~mm}$ posterior to the bregma. VEPs were recorded using a PUREC (Mayo Corp., Aichi, Japan). First, the rats were anaesthetized with intramuscular ketamine $(35 \mathrm{mg} / \mathrm{kg})$ and xylazine $(3.5 \mathrm{mg} / \mathrm{kg})$, and then, their pupils were dilated with $0.5 \%$ tropicamide and $0.5 \%$ phenylephrine hydrochloride. Photic stimuli of various intensities were generated using different colour (wavelengths: 468, 525 and $640 \mathrm{~nm}$ ) LEDs (VE-LED; ACrux Inc.) and applied for $10 \mathrm{~ms}$ with a frequency of $1 \mathrm{~Hz}$. The high- and low-pass filters were set at 3 and $500 \mathrm{~Hz}$, respectively. VEP responses were consecutively measured 200 times, and the collected response waveforms were averaged.

\section{Preparation of retinal sections and whole-mounted retinas}

Rat eyes were fixed overnight at $4^{\circ} \mathrm{C}$ in $4 \%$ paraformaldehyde prepared in phosphate-buffered saline (PBS; Fujifilm Wako Pure Chemical, Osaka). The eyes were rinsed with PBS, and the anterior parts, such as the cornea, iris, and lens, were removed. For preparing retinal sections, the eyecups were immersed in 10\%, 20\% and 30\% sucrose in PBS and embedded in optimal cutting temperature compound (Sakura, Tokyo, Japan) using a Histotech Pino system (Sakura, Tokyo, Japan). Cryosections $(12 \mu \mathrm{m})$ of the tissue were mounted on slides and air-dried. Whole-mounted retinal specimens were prepared by detaching the retinas from the eyecups and immunostaining them with an anti-NeuN antibody (MAB377; Merk Millipore, Tokyo, Japan).

\section{Immunohistochemistry}

Retinal sections were washed with PBS containing $0.3 \%$ Triton X-100 $(0.3 \%$ Tx-PBS) and treated for 10 min with 3\% Tx-PBS. Sections were blocked with $3 \%$ normal goat serum (Funakoshi, Tokyo) and 1\% BSA in 0.3\% Tx-PBS for $30 \mathrm{~min}$ at room temperature $\left(20-25^{\circ} \mathrm{C}\right)$ and incubated with ALEXA FLUOR 594-conjugated anti-NeuN polyclonal antibody $(2 \mu \mathrm{g} / \mathrm{mL}$; Cat No. bs1613R-A594, Bioss, MA, USA) overnight in a moist chamber at $4{ }^{\circ} \mathrm{C}$. After the slides were washed, coverslips were mounted with DAPI fluoromount$\mathrm{G}^{\star}$ (Cosmo-Bio, Tokyo, Japan). Retinas were treated with hyaluronidase (300 units $/ \mathrm{mL}$; Cat No. H3884, Merk, Tokyo, Japan) for $2 \mathrm{~h}$ at room temperature. After the retinas were washed with $0.5 \%$ Triton X-100 prepared in PBS (0.5\% Tx-PBS), they were incubated with ALEXA FLUOR ${ }^{\circ}$ 594-conjugated anti-NeuN polyclonal antibody $(5 \mu \mathrm{g} / \mathrm{mL})$ diluted in PBS containing $2 \%$ normal goat serum and $2 \%$ Triton $X-100$ overnight at room temperature. After retinas were washed with $0.5 \% \mathrm{Tx}-\mathrm{PBS}$, they were flatmounted on slides and covered with fluoromount- $G^{\oplus}$ (Cosmo-Bio). The sections and flat-mounted retinas were observed under a fluorescence microscope (Carl Zeiss, Tokyo, Japan).

\section{Behavioural assessment}

The spatial vision of an ex3mV1CO-expressing rat was measured by its optomotor response. We used a virtual optomotor system that was produced according to a sine wave function with the variable frequency with a stimulus of colour stripes over a black background previously described $^{16}$ with slight modification. We used a liquid crystal display instead of the projector that was previously used because ex $3 \mathrm{mV} 1 \mathrm{Co}$ has a higher sensitivity than mVChR1. The maximum intensity reached 103 lux of white light at the platform. The luminance values at the centre of the platform were 30, 40 and 36 lux when the colour was set to blue, green and red, respectively. Each wavelength displayed on the screen was measured by using an illumination metre (LX-1128SD; Mother Toll, Nagano, Japan). The experimenter waited until the rat stopped moving, and then, a homogeneous grey stimulus was projected for $5 \mathrm{~s}$ on the screen before the presentation of each grating session. The grating session was displayed with an alternative direction every $5 \mathrm{~s}$, and the session was displayed for $60 \mathrm{~s}$. An experimenter assessed whether the animals tracked the rotation by monitoring the head movement and presented the rotating stimulus simultaneously on the video camera monitor. If the movement was ambiguous, the same grating session was presented again. All behavioural tests were double-blinded and performed during the first few hours of the animals' light cycle (lights on at 8:00 AM).

\section{Statistical analysis}

Statistical analyses for in vitro experiments were performed using Sigma Plot ver. 12.3 (Hulinks, Tokyo, Japan) and MATLAB R2021a (The MathWorks, Natick, MA, USA). The statistical methods used were Tukey's multiple comparison test, Dunnett's multiple comparison test, Student's $t$ test, and one-way ANCOVA. A $p$-value $<0.05$ was considered significant.

\section{Molecular dynamics simulations}

The initial structural coordinates of ex3mV1 and ex3mV1Co proteins were determined based on the ground-state crystal structure of channelrhodopsin derived from PDB: 4yzi), using the homology modelling server SWISS-MODEL (https://swissmodel.expasy.org/). The model structure contains the protein dimer, covalently bound retinal chromophores, 5 oleic acids and 19 water molecules per protein monomer.

The potential energy function was set as follows: the consistent-valence forcefield force field was used for proteins and oleic acid, while the extensible and systematic force field ESFF was used for polyene chains (retinal). The following protocol was employed to perform an MD simulation: a simulation system was geometrically optimised by performing a 5000-step conjugate gradient energy minimisation. We fixed heavy atoms of proteins, as well as oleic acids and water molecules degraded in the crystal structure, during geometry optimisation. An equilibration calculation was performed by progressively decreasing position constraints in $200 \mathrm{ps}$ MD simulations, starting from a force constant of 25-10 and $1 \mathrm{kcal} / \mathrm{mol} \AA$, respectively. A 1 -ns simulation was then performed at a constant temperature of $298 \mathrm{~K}$ in the National Variety Trial ensemble, without the position constraint. An MD simulation was performed using the Discover/Insight II software package (Dassault Systemes BIOVIA, Discovery Studio Modelling Environment, Release, 2017, San Diego: Dassault Systemes, 2016). The time step was integrated at $1 \mathrm{fs}$, and the cut-off of the short-range interaction was $9.5 \AA$. The Discovery Studio 4.5 software package was used to manipulate molecular graphics and perform simulation analysis.

\section{Reporting summary}

Further information on research design is available in the Nature Research Reporting Summary linked to this article. 


\section{DATA AVAILABILITY}

All data supporting the findings of this study are available from the corresponding authors upon reasonable request.

Received: 23 February 2021; Accepted: 29 September 2021; Published online: 14 October 2021

\section{REFERENCES}

1. Nagel, G. et al. Channelrhodopsin-2, a directly light-gated cation-selective membrane channel. Proc. Natl Acad. Sci. USA 100, 13940-13945 (2003).

2. Nagel, G. et al. Channelrhodopsins: directly light-gated cation channels. Biochem. Soc. Trans. 33, 863-866 (2005).

3. Boyden, E. S., Zhang, F., Bamberg, E., Nagel, G. \& Deisseroth, K. Millisecondtimescale, genetically targeted optical control of neural activity. Nat. Neurosci. 8, 1263-1268 (2005).

4. Nagel, G. et al. Light activation of channelrhodopsin-2 in excitable cells of Caenorhabditis elegans triggers rapid behavioral responses. Curr. Biol. 15, 2279-2284 (2005).

5. Han, X. et al. Millisecond-timescale optical control of neural dynamics in the nonhuman primate brain. Neuron 62, 191-198 (2009).

6. Osawa, S. et al. Optogenetically induced seizure and the longitudinal hippocampal network dynamics. PLOS ONE 8, e60928 (2013).

7. Roy, D. S. et al. Memory retrieval by activating engram cells in mouse models of early Alzheimer's disease. Nature 531, 508-512 (2016).

8. Tomita, H., Sugano, E., Isago, H. \& Tamai, M. Channelrhodopsins provide a breakthrough insight into strategies for curing blindness. J. Genet. 88, 409-415 (2009).

9. $\mathrm{Bi}, \mathrm{A}$. et al. Ectopic expression of a microbial-type rhodopsin restores visual responses in mice with photoreceptor degeneration. Neuron 50, 23-33 (2006).

10. Tomita, H. et al. Visual properties of transgenic rats harboring the channelrhodopsin-2 gene regulated by the thy-1.2 promoter. PLOS ONE 4, e7679 (2009).

11. Tomita, H. et al. Channelrhodopsin-2 gene transduced into retinal ganglion cells restores functional vision in genetically blind rats. Exp. Eye Res. 90, 429-436 (2010).

12. Tomita, H. et al. Restoration of visual response in aged dystrophic RCS rats using AAV-mediated channelopsin-2 gene transfer. Invest. Ophthalmol. Vis. Sci. 48, 3821-3826 (2007).

13. Sugano, E. et al. Immune responses to adeno-associated virus type 2 encoding channelrhodopsin-2 in a genetically blind rat model for gene therapy. Gene Ther. 18, 266-274 (2011).

14. Ivanova, E., Hwang, G. S., Pan, Z. H. \& Troilo, D. Evaluation of AAV-mediated expression of Chop2-GFP in the marmoset retina. Invest. Ophthalmol. Vis. Sci. 51, 5288-5296 (2010).

15. Sahel, J. A., et al. Partial recovery of visual function in a blind patient after optogenetic therapy. Nat. Med. https://doi.org/10.1038/s41591-021-01351-4 (2021).

16. Tomita, H. et al. Restoration of the majority of the visual spectrum by using modified Volvox channelrhodopsin-1. Mol. Ther. 22, 1434-1440 (2014).

17. Sugano, E. et al. Local and systemic responses following intravitreous injection of AAV2-encoded modified Volvox channelrhodopsin-1 in a genetically blind rat model. Gene Ther. 23, 158-166 (2016).

18. Sato, M. et al. Visual responses of photoreceptor-degenerated rats expressing two different types of channelrhodopsin genes. Sci. Rep. 7, 41210 (2017).

19. Berndt, A. et al. High-efficiency channelrhodopsins for fast neuronal stimulation at low light levels. Proc. Natl Acad. Sci. USA 108, 7595-7600 (2011).

20. Lin, J. Y., Lin, M. Z., Steinbach, P. \& Tsien, R. Y. Characterization of engineered channelrhodopsin variants with improved properties and kinetics. Biophys. J. 96, 1803-1814 (2009).

21. Kato, H. E. et al. Crystal structure of the channelrhodopsin light-gated cation channel. Nature 482, 369-374 (2012).

22. Wang, H. et al. Molecular determinants differentiating photocurrent properties of two channelrhodopsins from chlamydomonas. J. Biol. Chem. 284, 5685-5696 (2009).

23. Bedbrook, C. N. et al. Structure-guided SCHEMA recombination generates diverse chimeric channelrhodopsins. Proc. Natl Acad. Sci. USA 114, E2624-E2633 (2017).

24. Mattis, J. et al. Principles for applying optogenetic tools derived from direct comparative analysis of microbial opsins. Nat. Methods 9, 159-172 (2011).

25. Schild, L. C. \& Glauser, D. A. Dual color neural activation and behavior control with Chrimson and CoChR in Caenorhabditis elegans. Genetics 200, 1029-1034 (2015).

26. Shemesh, O. A. et al. Temporally precise single-cell-resolution optogenetics. Nat. Neurosci. 20, 1796-1806 (2017).
27. Ganjawala, T. H., Lu, Q., Fenner, M. D., Abrams, G. W. \& Pan, Z. H. Improved CoChR variants restore visual acuity and contrast sensitivity in a mouse model of blindness under ambient light conditions. Mol. Ther. 27, 1195-1205 (2019).

28. Ernst, O. P. et al. Photoactivation of channelrhodopsin. J. Biol. Chem. 283, 1637-1643 (2008).

29. Hegemann, P., Ehlenbeck, S. \& Gradmann, D. Multiple photocycles of channelrhodopsin. Biophys. J. 89, 3911-3918 (2005).

30. Ernst, O. P. et al. Microbial and animal rhodopsins: structures, functions, and molecular mechanisms. Chem. Rev. 114, 126-163 (2014).

31. Stehfest, K., Ritter, E., Berndt, A., Bartl, F. \& Hegemann, P. The branched photocycle of the slow-cycling channelrhodopsin-2 mutant C128T. J. Mol. Biol. 398, 690-702 (2010).

32. Govorunova, E. G., Sineshchekov, O. A. \& Spudich, J. L. Structurally distinct cation channelrhodopsins from cryptophyte algae. Biophys. J. 110, 2302-2304 (2016).

33. Hososhima, S., Shigemura, S., Kandori, H. \& Tsunoda, S. P. Novel optogenetics tool: Gt_CCR4, a light-gated cation channel with high reactivity to weak light. Biophys. Rev. 12, 453-459 (2020).

34. Wu, B. M., Leng, T. D., Inoue, K., Li, J. \& Xiong, Z. G. Effect of redox-modifying agents on the activity of channelrhodopsin-2. CNS Neurosci. Ther. 23, 216-221 (2017).

35. Gaiko, O. \& Dempski, R. E. Transmembrane domain three contributes to the ion conductance pathway of channelrhodopsin-2. Biophys. J. 104, 1230-1237 (2013).

36. Takemoto, M. et al. Molecular dynamics of channelrhodopsin at the early stages of channel opening. PLOS ONE 10, e0131094 (2015).

37. Doi, S., Tsukamoto, T., Yoshizawa, S. \& Sudo, Y. An inhibitory role of Arg-84 in anion channelrhodopsin-2 expressed in Escherichia coli. Sci. Rep. 7, 41879 (2017).

38. Kojima, K. et al. Mutational analysis of the conserved carboxylates of anion channelrhodopsin-2 (ACR2) expressed in Escherichia coli and their roles in anion transport. Biophys. Physicobiol. 15, 179-188 (2018).

39. Wang, Z. et al. Differentiation of neuronal cells from NIH/3T3 fibroblasts under defined conditions. Dev. Growth Differ. 53, 357-365 (2011).

40. Tinsley, J. N. et al. Direct detection of a single photon by humans. Nat. Commun. 7, 12172 (2016)

41. Brezovsky, J., Kozlikova, B. \& Damborsky, J. Computational analysis of protein tunnels and channels. Methods Mol. Biol. 1685, 25-42 (2018).

42. Kato, H. E. \& Nureki, O. Crystal structure of channelrhodopsin, a light-gated cation channel-all cations lead through the monomer. Biophysics 9, 57-61 (2013).

43. Klapoetke, N. C. et al. Independent optical excitation of distinct neural populations. Nat. Methods 11, 338-346 (2014).

44. Isago, H. et al. Age-dependent differences in recovered visual responses in Royal College of Surgeons rats transduced with the Channelrhodopsin-2 gene. J. Mol. Neurosci. 46, 393-400 (2012).

45. Graham, F. L. \& van der Eb, A. J. A new technique for the assay of infectivity of human adenovirus 5 DNA. Virology 52, 456-467 (1973).

46. Watanabe, Y. et al. Kinetic profiles of photocurrents in cells expressing two types of channelrhodopsin genes. Biochem. Biophys. Res. Commun. 496, 814-819 (2018).

47. LaVail, M. M. \& Battelle, B. A. Influence of eye pigmentation and light deprivation on inherited retinal dystrophy in the rat. Exp. Eye Res. 21, 167-192 (1975).

48. Sugano, E., Tomita, H., Ishiguro, S., Abe, T. \& Tamai, M. Establishment of effective methods for transducing genes into iris pigment epithelial cells by using adenoassociated virus type 2. Invest. Ophthalmol. Vis. Sci. 46, 3341-3348 (2005).

\section{ACKNOWLEDGEMENTS}

The authors thank Mses. Yoko Takahashi, Misao Enomoto, Miho Sato, and Takako Kido of the Laboratory of Visual Neuroscience for maintaining the RCS rats and HEK293 cells used in this study. This work was supported by Grants-in-Aid for Scientific Research from the Ministry of Education, Culture, Sports, Science and Technology, Japan (Nos. 19H03807, 19K09945, 18K09433 and 17H06330).

\section{AUTHOR CONTRIBUTIONS}

H.T. and E.S. planned the research; Y.W., H.T., K.T., E.S. and A.H. performed the experiments; Y.W., K.T. and A.H. analysed the data; T.S., T.F., T.S. and T.O. provided the technical support; Y.W. prepared the original paper draft; H.T. and E.S. reviewed and edited the paper; H.T. was responsible for visualisation; H.T., E.S. and K.T. procured funding for the study.

\section{COMPETING INTERESTS}

The authors declare no competing interests. 


\section{ADDITIONAL INFORMATION}

Supplementary information The online version contains supplementary material available at https://doi.org/10.1038/s41536-021-00177-5.

Correspondence and requests for materials should be addressed to Eriko Sugano or Hiroshi Tomita.

Reprints and permission information is available at http://www.nature.com/ reprints

Publisher's note Springer Nature remains neutral with regard to jurisdictional claims in published maps and institutional affiliations.
Open Access This article is licensed under a Creative Commons Attribution 4.0 International License, which permits use, sharing, adaptation, distribution and reproduction in any medium or format, as long as you give appropriate credit to the original author(s) and the source, provide a link to the Creative Commons license, and indicate if changes were made. The images or other third party material in this article are included in the article's Creative Commons license, unless indicated otherwise in a credit line to the material. If material is not included in the article's Creative Commons license and your intended use is not permitted by statutory regulation or exceeds the permitted use, you will need to obtain permission directly from the copyright holder. To view a copy of this license, visit http://creativecommons.org/licenses/by/4.0/.

(c) The Author(s) 2021 